\title{
Effects of Sulfate Position on Heparin Octasaccharide Binding to CCL2 Examined by Tandem Mass Spectrometry
}

\author{
Matt D. Sweeney, Yonghao Yu, and Julie A. Leary \\ Genome Center, Department of Chemistry, University of California, Davis, California, USA
}

\begin{abstract}
Chemokine-glycosaminoglycan (GAG) interactions have been shown to be essential for in vivo chemokine signaling, which functions in such diverse processes as inflammation, development, and cancer metastasis. Despite the importance of these interactions, the saccharide sequence dependency of chemokine-GAG interactions is poorly understood. In a recent study, FT-ICR mass spectrometry was used to show that the chemokine CCL2 (monocyte chemoattractant protein 1) binds only to the 11- and 12-sulfated components of a heparin octasaccharide library. Although the exact structure of the fully sulfated, 12-sulfated octasaccharide is known, the 11-sulfated species could have a number of sulfated disaccharide sequences. In the current study, the composition of the 11-sulfated heparin octasaccharides, as well as the composition of CCL2 affinity purified 11-sulfated heparin octasaccharides, were examined by tandem MS. Of the three possible singly desulfated disaccharides, one species, III-S, is enriched by CCL2 affinity purification, indicating that the 11-sulfated heparin octasaccharides containing this disaccharide are preferentially bound to CCL2. These data suggest that $2-\mathrm{O}$ and $\mathrm{N}$ sulfation of heparin may be of greater importance to CCL2-heparin binding than 6-O sulfation. (J Am Soc Mass Spectrom 2006, 17, 1114-1119) () 2006 American Society for Mass Spectrometry
\end{abstract}

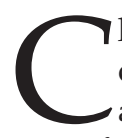
hemokine mediated signaling depends not only on the ability of a chemokine to complex with and activate a receptor, but also on the formation of a chemokine concentration gradient that directs chemotaxis. Chemokines diffuse away from the site of secretion until they bind cell-surface glycosaminoglycans (GAGs) resulting in the formation of a chemotactic gradient that can be followed by certain motile cell types $[1,2]$. While mutant chemokines that are unable to bind GAGs can still bind receptor and signal in vitro, they are unable to recruit immune cells in vivo [3,4]. Despite the importance of GAG-chemokine binding, the role of the specific sequence of the GAG component is poorly understood.

The principle GAG ligand for inflammatory chemokines is cell-surface heparan sulfate [5]. Both heparan sulfate and the more highly sulfated heparin contain, upon enzymatic digestion of the polymers, 12 common disaccharide subunits, not including changes to epimerization or the 3-O sulfate isomers [6]. This results in a high degree of sequence complexity for even fairly short chains. In spite of this heterogeneity, GAG binding sequence specificity has been observed to various

Published online June 2, 2006

Address reprint requests to Dr. J. A. Leary, Genome Center, Department of Chemistry, Room 1300, University of California, 1 Shields Avenue, Davis, CA 95616, USA. E-mail: jaleary@ucdavis.edu extents in binding to proteins such as the members of the FGF family [7-9]. The rare 3-O sulfation further increases the possible complexity of heparinoids, and has been shown to be essential in heparin binding to antithrombin III $[10,11]$ and in herpes simplex virus 1 infection [12].

As most inflammatory chemokines are positively charged and heparin and heparan sulfate are negatively charged, ionic and electrostatic interactions are clearly important in chemokine-GAG binding, however it is unclear what role GAG sulfate positioning plays in possible selectivity of chemokine binding sites. In a recent study, it was shown that the five chemokine ligands of chemokine receptor 2 (CCR2) bind to only the highly sulfated components of a heparin octasaccharide library [13]. One of these chemokines, CCL2 (CC chemokine ligand 2), was shown to bind only to heparin octasaccharide that is either fully sulfated (12sulfate) or singly unsulfated (11-sulfate). This finding was surprising in that heparan sulfate, the principle GAG ligand for CCL2, is not highly sulfated, though local patches of high sulfation (S-domains) exist connected by less sulfated regions in the chain [14]. If such highly sulfated stretches of GAG are the binding sites for CCL2, the possible sequence complexity of heparan sulfate in these epitopes decreases dramatically. Studies of the binding of heparan sulfate to the chemokines interleukin-8 (CXCL8), platelet factor 4 (CXCL4), and 
macrophage inflammatory protein $1 \alpha$ (CCL3) all identified chemokine binding sites consisting of fairly large sequences of 14-24 saccharides [15-17]. These binding sites appear to consist of two S-domains connected by a less sulfated region.

The heparin octasaccharide which binds to CCL2 is structurally similar to heparan sulfate S-domain, and by examining the determinates of CCL2 binding to highly sulfated sequences of heparin, information on the relative importance of positional sulfation in CCL2 binding to highly sulfated heparan sulfate may be ascertained. The octasaccharide in our library was generated by heparinase I digestion, which cleaves heparin only after $\mathrm{N}$-sulfated glucosamine and before 2-O sulfated iduronate or 2-O sulfated glucoronate, leaving $\mathrm{N}$ - and 2-O sulfated terminal sugars [18, 19]. Given this constraint, the 11-sulfated octasaccharide can be unsulfated at only ten possible sites, resulting in only ten possible disaccharide sulfation sequences. To investigate the possibility of sequence selectivity by CCL2 in heparin binding, we purified the 11-sulfated octasaccharides, screened these octasaccharides for CCL2 binding, and determined the disaccharide composition of both the initial 11-sulfated octasaccharide library and of the CCL2 affinity enriched 11-sulfated octasaccharide using an ESI tandem mass spectrometry.

Sensitivity and speed make mass spectrometry an attractive approach for analysis of sulfated glycosaminoglycans. One issue of concern is the liability of the sulfate groups on these molecules, however solvent conditions can be manipulated to minimize this loss [20, 21]. Complete enzymatic digestion to disaccharide subunits for compositional analysis overcomes the problem of sulfate loss, but has the disadvantage that any information about uronic acid epimerization or sequence is lost [6, 22]. Methods using partial digestion, isotopic labeling, and LC-MS have been developed [23-26], and these studies have helped to propel forward the field of GAG analysis. It is import to note, however, that these methods are still somewhat sensitive to glycosaminoglycan length and heterogeneity.

\section{Materials and Methods}

\section{Reagents and Instrumentation}

Heparin octassacharide library was purchased from Dextra (Reading, UK). CarboPac PA1 ion-exchange column was purchased from Dionex (Sunnyvale, CA). High-pressure liquid chromatography (HPLC) grade solvents were purchased from Fisher (Santa Clara, CA). SAX HPLC was purchased from Waters (Milford, MA). Heparin desalting was performed using $1 \mathrm{kDa}$ DispoBiodialyzers (The Nest Group, Southborough, MA). Oasis HLB C18 column was purchased from Waters (Milford, MA). Heparinase I, II, and III were purchased from Seikagaku (East Falmouth, MA). Disaccharide standards were purchased from Sigma-Aldich (St. Louis, MO). Heparin disaccharide stock solutions were prepared at concentrations of $2 \mathrm{mM}$ on the basis of their absorbance at $232 \mathrm{~nm}$ in $0.03 \mathrm{M} \mathrm{HCl} \varepsilon_{232}=5500 \mathrm{M}^{-1}$ $\mathrm{cm}^{-1}$ ) [27].

High-resolution mass spectra were acquired on a Bruker APEX II 7-tesla FT-ICR (Billerica, MA) mass spectrometer that was equipped with an Apollo (Bruker) electrospray ionization (ESI) source. Samples in a solvent of 1:1 $\mathrm{MeOH} / \mathrm{H}_{2} \mathrm{O}$ with $20 \mathrm{mM} \mathrm{NH} 4 \mathrm{OH}$ were infused into the mass spectrometer at $1 \mu \mathrm{L} / \mathrm{min}$ using a syringe pump (Harvard Apparatus, Holliston, $\mathrm{MA}$ ). Nitrogen at 50 psi was used as the nebulizing gas, while capillary exit voltage was adjusted to $40 \mathrm{~V}$ for ion desolvation. Ions were externally accumulated in a radio frequency-only hexapole for $0.5 \mathrm{~s}$. Ions were trapped using gated trapping and detected using negative polarity. All the data were acquired and processed using Xmass version 6.0.0 (Bruker).

Mass spectra for compositional analysis were obtained by using an ESI source on an LTQ MS (ThermoFinnigan, San Jose, CA). Disaccharide standards were sprayed at a concentration of $20 \mathrm{pmol} / \mu \mathrm{L}$ for each disaccharide ( $20 \mathrm{pmol} / \mu \mathrm{L}$ total disaccharide concentration for experimental samples) in a $1: 1 \mathrm{MeOH} / \mathrm{H}_{2} \mathrm{O}$ solution containing $10 \mathrm{mM} \mathrm{NH}{ }_{4} \mathrm{OH}, 2 \mathrm{mM} \mathrm{NH}_{4} \mathrm{OAc}$, $0.2 \mathrm{mM} \mathrm{Ca}(\mathrm{OAc})_{2}$, and $5 \mathrm{pmol} / \mu \mathrm{L}$ internal standard I-P. Samples were introduced by flow injection at 20 $\mu \mathrm{L} / \mathrm{min}$ in $1: 1 \mathrm{MeOH} / \mathrm{H}_{2} \mathrm{O}$ using a syringe pump (Harvard Apparatus). Spectra were obtained in negative ion mode by using a spray voltage of $3.8 \mathrm{kV}$ and a capillary temperature of $230^{\circ} \mathrm{C}$ for all experiments. For MS $^{2}$ experiments, a 4 Da isolation width was used for precursor ion selection, and the ion was activated at $21 \%$ normalized collision energy for $100 \mathrm{~ms}$, and the $q_{z}$ value was maintained at 0.250 . Each mass spectrum obtained contains an average of 10 scans. Xcalibur, version 1.4 (ThermoFinnigan), was used for data acquisition.

\section{Preparation of 11-Sulfated Heparin Octasaccharide}

Heparin octasaccharide with 11 sulfates was isolated from the heparin octasaccharide mixture by using strong anion-exchange HPLC. Approximately $400 \mu \mathrm{g}$ of an octasaccharide library was injected onto a CarboPac PA1 column and SAX HPLC was performed at a flow rate of $1 \mathrm{~mL} / \mathrm{min}$ using Solvent $\mathrm{A}\left(\mathrm{H}_{2} \mathrm{O}, \mathrm{pH} 3.5\right)$ and Solvent B (3 M NaCl, pH 3.5). A typical gradient consisted of: (1) 1-11 min, $0 \%$ B, (2) $11-110 \mathrm{~min}, 0-100 \%$ $\mathrm{B}$, and (3) 111-120 min, 0\% B. Chromatograms were recorded by monitoring the UV absorbance at $232 \mathrm{~nm}$. Fractions corresponding to 11-sulfated octasaccharide were collected, lyophilized and desalted.

\section{Preparation of CCL2}

CCL2 was expressed, purified, and processed as previously described [28]. Briefly, CCL2 was expressed using a codon-optimized construct in TAP302 cells. Soluble CCL2 was purified by ion-exchange chromatography 


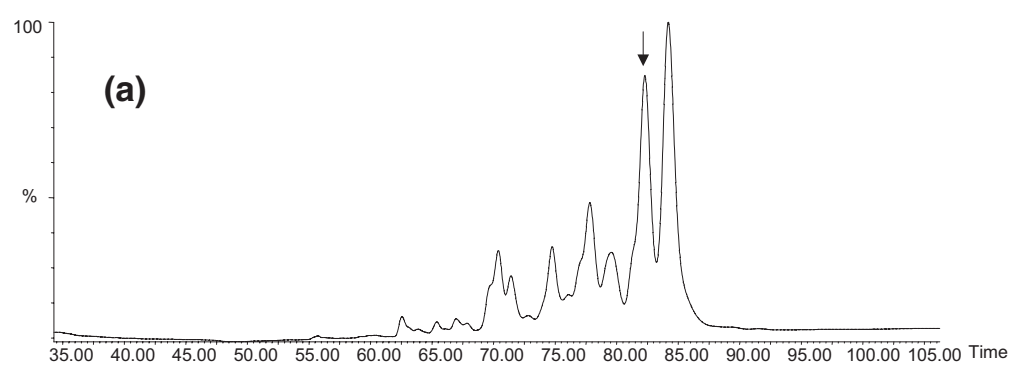

(b) $\left[\mathrm{Octa} / 11 \mathrm{SO}_{3}\right]^{6-}$

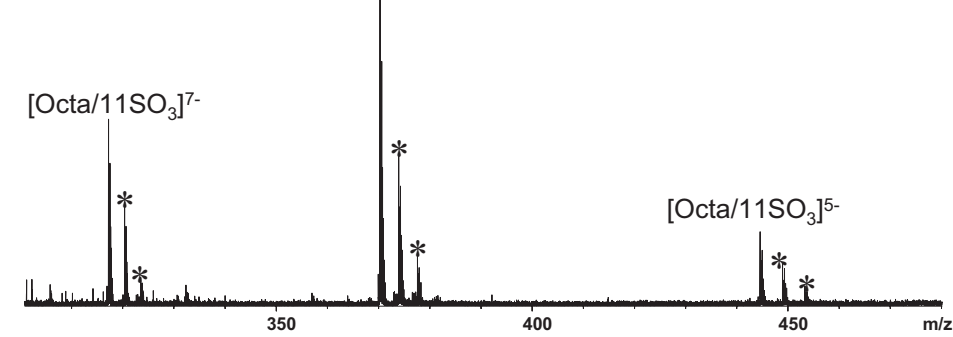

Figure 1. Preparation of heparin octasaccharide modified with 11 sulfates. (a) Chromatogram of SAX HPLC separation of heparin octasaccharide library. Fraction indicated by the arrow was collected and desalted. (b) ESI-FTICR mass spectra of the fraction corresponding to 11-sulfated heparin octasaccharide $\left(\mathrm{Octa} / 11 \mathrm{SO}_{3}\right)$. Asterisks represents the sodium adducts.

followed by reverse-phase high-performance liquid chromatography (RP-HPLC). The N-terminal methionine was removed by aminopeptidase, leaving authentic human CCL2 that was then subject to re-purification by RP-HPLC.

\section{CCL2 Affinity Enrichment of 11-Sulfated Octasaccharide}

Octasaccharide was enriched by incubating $40 \mu \mathrm{M}$ CCL2 with $200 \mu \mathrm{M}$ 11-sulfate octasaccharide in $100 \mu \mathrm{L}$ of $100 \mathrm{mM} \mathrm{NH}_{4} \mathrm{OAc}$ solution ( $\mathrm{pH}$ 6.8). The solution was applied to an Oasis solid-phase extraction (SPE) cartridge (Waters, Milford, MA) that had been previously conditioned using $1 \mathrm{~mL}$ of methanol and then $1 \mathrm{~mL}$ of $\mathrm{H}_{2} \mathrm{O}$. The solid-phase SPE cartridge was flushed three times, each using $1 \mathrm{~mL} 200 \mathrm{mM} \mathrm{NH}_{4} \mathrm{OAc}$ solution ( $\mathrm{pH}$ 6.8) and then eluted by $1 \mathrm{~mL} 760 \mathrm{mM} \mathrm{NH}_{4} \mathrm{OAc}(\mathrm{pH}$ 6.8). The three washing fractions and the final elution faction were collected and desalted by dialyzing against water using a dialyzer with a molecular weight cutoff (MWCO) of $1 \mathrm{kDa}$.

\section{Compositional Analysis of Affinity Purified Heparin Octasaccharide}

Enzymatic digestion of heparin octasaccharide to constituent disaccharides was carried out with $5 \mu \mathrm{g}$ heparin in $20 \mu \mathrm{L}$ of $20 \mathrm{mM}$ ammonium acetate buffer, $\mathrm{pH}$ $7.5,2 \mathrm{mM}$ calcium acetate, and 0.001 unit each of heparinase I, heparinase II, and heparinase III, which was incubated at $37^{\circ} \mathrm{C}$ for $16 \mathrm{~h}$. The reaction was quenched yielding a final solution containing 20 $\mathrm{pmol} / \mu \mathrm{L}$ heparin disaccharide, 1:1 $\mathrm{MeOH} / \mathrm{H}_{2} \mathrm{O}, 5$ $\mathrm{pmol} / \mu \mathrm{L}$ internal standard I-P, and $10 \mathrm{mM}$ ammonium hydroxide. Samples were then analyzed using a combination of ESI-MS and MS-MS on an LTQ mass spectrometer. An $\mathrm{MS}^{1}$ step is used to quantify the nonisomeric disaccharide and the total combined concentration of isomeric disaccharides by comparing peak intensities with the peak intensity of the internal standard, I-P. The second $\mathrm{MS}^{2}$ step is then used to determine the relative concentrations of each of the $\mathrm{m} / \mathrm{z}$ 247.7 isomers by observing ratios of diagnostic ions that are formed by collision-induced dissociation of the parent ions. Both experimental and control samples were analyzed in triplicate: i.e., each sample work-up and analysis was preformed three times on three different days. Data were processed using QualBrowser (ThermoFinnigan) and Excel (Microsoft). This method has been used previously to quantify complicated mixtures of 12 heparin disaccharides, and gives results comparable to HPLC analysis [6, 29].

\section{Results and Discussion}

To obtain the 11-sulfated heparin octasaccharide, it was necessary to fractionate the heparin octasaccharide library on the basis of charge. The octasaccharide library, which contained between 8 and 12 sulfates per molecule, was applied to a strong anion exchange column (CarboPac PA1) and eluted using a linear $\mathrm{NaCl}$ gradient, yielding well resolved peaks corresponding to the extent of sulfation (Figure 1a). The penultimate peak to elute was collected, desalted extensively, and analyzed by FT-ICR mass spectrometry, which yielded an ion at 


\section{Heparin Disaccharides}

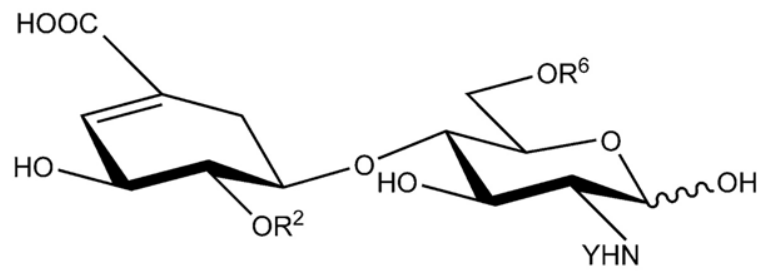

\begin{tabular}{lllllll}
\multicolumn{2}{c}{ Disaccharide } & $\mathrm{R}^{2}$ & $\mathrm{R}^{6}$ & $\mathrm{Y}$ & $\mathrm{MS}^{1} \mathrm{~m} / z$ & $\mathrm{MS}^{2} \mathrm{~m} / \mathrm{z}$ \\
$\mathrm{III}-\mathrm{S}$ & $\triangle \mathrm{UA2S} \rightarrow$ GlcNS & $\mathrm{SO}_{3}$ & $\mathrm{H}$ & $\mathrm{SO}_{3}$ & $247.7^{2-}$ & $398.0^{1-}, 97.0^{1-}$ \\
$\mathrm{II}-\mathrm{S}$ & $\triangle \mathrm{UA} \rightarrow$ GlcNS6S & $\mathrm{H}$ & $\mathrm{SO}_{3}$ & $\mathrm{SO}_{3}$ & $247.7^{2-}$ & $338.0^{1--}, 168.6^{1-}$ \\
$\mathrm{I}-\mathrm{H}$ & $\triangle \mathrm{UA2S} \rightarrow$ GlcN6S & $\mathrm{SO}_{3}$ & $\mathrm{SO}_{3}$ & $\mathrm{H}$ & $247.7^{2-}$ & $218.1^{2-}$ \\
$\mathrm{I}-\mathrm{S}$ & $\triangle \mathrm{UA} 2 \mathrm{~S} \rightarrow$ GlcNS6S & $\mathrm{SO}_{3}$ & $\mathrm{SO}_{3}$ & $\mathrm{SO}_{3}$ & $191.4^{3-}$ & N/A
\end{tabular}

Figure 2. Variable disaccharide repeat of heparin and heparan sulfate. The four possible disaccharide components of our 11sulfated octasaccharide are indicated, along with major $\mathrm{MS}^{1}$ ion and $\mathrm{MS}^{2}$ diagnostic ion(s).

$\mathrm{m} / \mathrm{z}$ of 370.3143 corresponding to the 6-charge state of a heparin octasaccharide with 11 sulfates and no acetyl groups (Figure 1b, FT-ICR spectra for other fractions not shown). The theoretical value for the 6-charge state is 370.3141 , and the difference averaged over the three charge states 5-, 6-, and 7- is $0.32 \mathrm{ppm}$. The measured molecular mass of this isomer is $2227.9284 \mathrm{Da}$ (theoretical 2227.9285). The high-resolution of the FT-ICR makes this accuracy possible.

CCL2 was incubated with the 11-sulfated octasaccharide isomers and subsequently bound to a hydrophobic column under low salt conditions. Samples of the load (11-sulfated octasaccharide) and eluate (11-sulfated octasaccharide eluted by high salt) from the CCL2 enrich-
Table 1. The disaccharide composition of each octasaccharide fraction as calculated by LTQ-MS analysis

\begin{tabular}{lcr}
\hline \multicolumn{2}{c}{ Calculated abundance of heparin disaccharide composition } \\
\hline \multicolumn{3}{c}{$\%$ Abundance } \\
\cline { 2 - 3 } Disaccharide & \multicolumn{2}{c}{ Load } \\
\hline \hline III-S & $8.86 \pm 3.38$ & Eluate \\
II-S & $85.73 \pm 5.44$ & $23.96 \pm 6.40$ \\
I-H & $5.33 \pm 3.46$ & $74.46 \pm 6.32$ \\
& & $1.50 \pm 0.86$
\end{tabular}

Each 11-sulfated octasaccharide contained three I-S fully sulfated disaccharides and one singly unsulfated III-S, II-S, or I-H disaccharide. Reported in this table is the percent abundance of these singly unsulfated disaccharides in each octasaccharide fraction. Results reflect triplicate experiments from affinity enrichment to MS-MS analysis; error is standard deviation between analyses of three datasets.

ment were extensively desalted, concentrated, and subjected to complete enzymatic digestion to their disaccharide constituents. These digested samples were then quenched and analyzed on an LTQ mass spectrometer for disaccharide composition by comparison of the diagnostic ions from the product ion spectra [29].

Standard mixes of known disaccharide composition and concentration were also analyzed by LTQ-MS as controls to monitor the identification and quantification procedure. Using diagnostic ions (Figure 2) from spectral data of the standard disaccharide mixes, response factors for each of the four possible disaccharides were calculated. Samples from the load and eluate fractions that were digested were then processed using these calculated response factors, and ratios of disaccharide concentration were determined [6]. The measured disaccharide composition is summarized in Table 1.
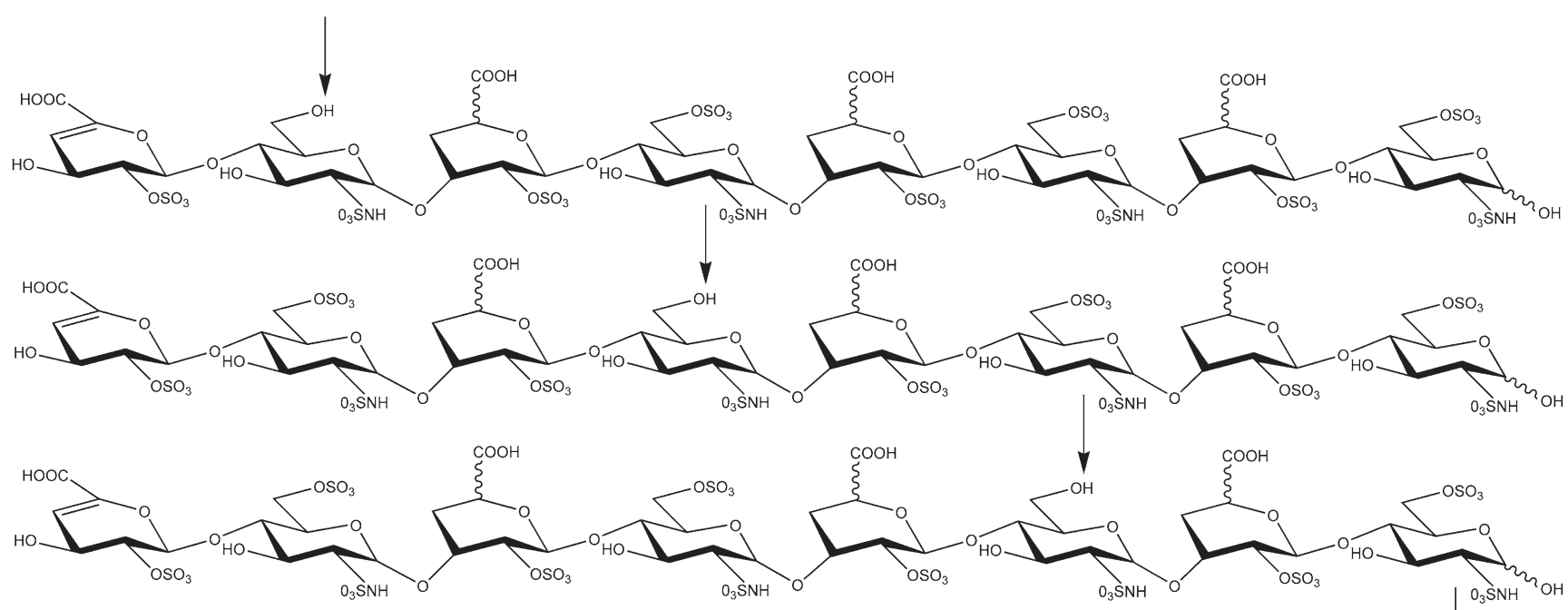

(1)
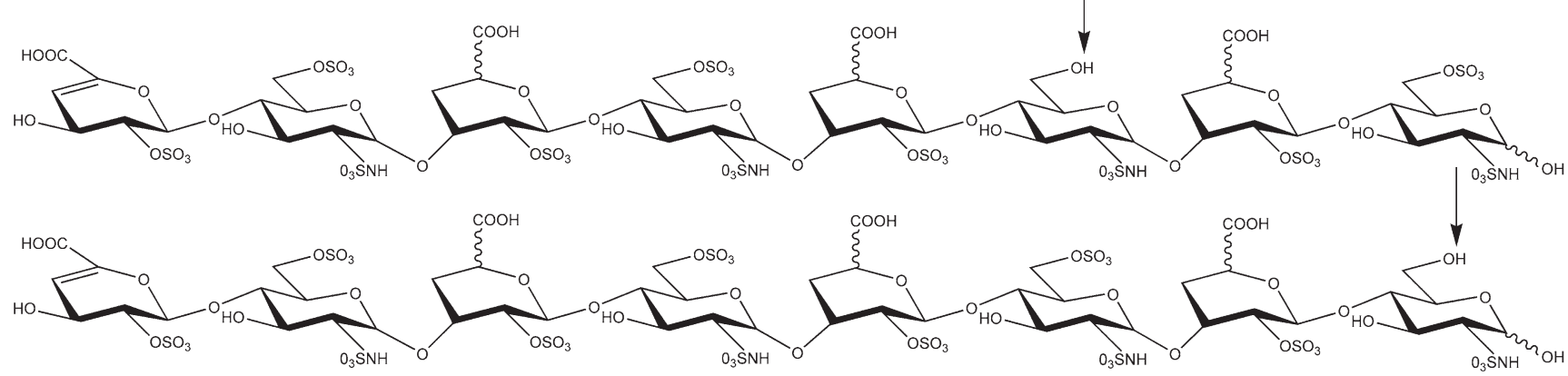

Figure 3. Structures of the four possible CCL-2 binding enriched heparin octasaccharide sequences. Arrows denote unsulfated positions. 
Our data indicate that the total percentage of disaccharide III-S is increased by a statistically significant amount by CCL2 affinity enrichment. This indicates that one or more of the four possible octasaccharide sulfation sequences containing a III-S disaccharide subunit are enriched by CCL2 binding (Figure 3, Table 1). It should be noted, however, that since our assay did not include the 3-O sulfated disaccharides, the disulfated version of this isomer is not excluded as a possible binder.

There are two possibilities for why this enrichment occurs. First, possibly an unsulfated 6-O position of III-S is forming a specific contact with CCL2, resulting in increased affinity between octasaccharide and chemokine. The other possibility is that loss of a 2-O (II-S) or $\mathrm{N}$ (I-H) sulfate will result in significantly weakened binding affinity between octasaccharide and CCL2 by disrupting an interaction with a 2-O or $\mathrm{N}$ sulfate group. Using the current assay, these hypotheses cannot be differentiated. An analysis comparing CCL2 binding to chemically synthesized heparin octasaccharides containing one III-S disaccharide in defined positions would be one approach to answering this question.

The heparan sulfate-binding site for several other chemokines has also been studied [14-16]. The heparan sulfate binding sites for these chemokines were all fairly large, in the range of 12-24 saccharide units. This size is consistent with GAG binding to more than one site on the chemokine dimer. Evidence in each publication was presented, which supports the model that two sulfated regions exist on each GAG molecule, linked by a $\mathrm{N}$ acetylated region of low sulfation. Not surprisingly, the basic chemokines CXCL8 and CXCL4 bound with greater affinity to more highly sulfated heparan sulfate, but this was also found to be true for the acidic chemokine CCL3. Some differences, other than the length of the heparan sulfate, were observed in the binding of these chemokines. CXCL8 showed little preference for $\mathrm{N}$ or $\mathrm{O}$ sulfate positioning, though at least one I-S disaccharide appears to be important for the interaction. CXCL4 bound 2-O sulfated regions preferably to 6-O sulfated regions of GAG, and while it did not have an absolute requirement of $\mathrm{N}$ sulfation to bind, high levels of $\mathrm{N}$ sulfation appear in the binding site. CCL3, which had the smallest GAG binding site of the three at 12-14 saccharides, had a binding site similar to CXCL4 but showed an absolute requirement for at least some 6-O sulfation to bind, whereas CXCL4 did not.

The heparin octasaccharides studied herein are substantially shorter and more sulfated than the commensurate heparan sulfate sequences that have been show to bind CXCL8, CXCL4, and CCL3. They mimic only one heparan sulfate S-domain and have no $\mathrm{N}$-acetylated linker region. However, the proposed models for heparan sulfate binding to these three chemokines all show one molecule of heparan sulfate bridging two GAG binding sites on the chemokines. The two binding site model is in agreement with the spacing of the GAG binding sites on the proposed dimer and tetramer structures of some chemokines. CCL2, however, forms a tetramer with one extended GAG binding site on each side $[30,3]$. Further, addition of heparin octasaccharide has been shown to promote formation of the CCL2 tetramer. As the GAG binding site of CCL2 is highly basic, any heparan sulfate chain that binds would have to be highly sulfated, suggesting that our heparin octasaccharide may not be very dissimilar to the actual heparan sulfate ligand.

\section{Conclusions}

Our data supports the hypothesis that the chemokine CCL2 preferentially binds highly sulfated heparin sequences, in particular those that contain at least one III-S disaccharide. Further studies are currently underway to identify specific heparan sulfate binders and to further probe the significance of positional isomers in CCL2 binding.

\section{Acknowledgments}

This research was supported by NIH grant GM 047356 to JAL. The authors thank Dr. Tracy Handel and Susan Crown for providing the construct for the CCL2 chemokine used in this study.

\section{References}

1. Rot, A. Endothelial cell binding of NAP-1/IL-8: Role in neutrophil emigration. Immunol. Today 1992, 13, 291-294.

2. Handel, T. M.; Johnson, Z.; Crown, S. E.; Lau, E. K.; Sweeney, M.; Proudfoot, A. E. Regulation of protein function by glycosaminoglycans-as exemplified by chemokines. Annu. Rev. Biochem. 2005, 74, 385-410.

3. Lau, E. K.; Paavola, C. D.; Johnson, Z.; Gaudry, J. P.; Geretti, E.; Borlat, F.; Kungl, A. J.; Proudfoot, A. E.; Handel, T. M. Identification of the glycosaminoglycan binding site of the CC chemokine, MCP-1: Iimplications for structure and function in vivo. J. Biol. Chem. 2004, 279, 22294-22305.

4. Proudfoot, A. E.; Handel, T. M.; Johnson, Z.; Lau, E. K.; LiWang, P.; Clark-Lewis, I.; Borlat, F.; Wells, T. N. C; Kosco-Vilbois, M. H. Glycosaminoglycan binding and oligomerization are essential for the in vivo activity of certain chemokines. Proc. Natl. Acad. Sci. U.S.A. 2003, 100, 1885-1890.

5. Middleton, J.; Neil, S.; Wintle, J.; Clark-Lewis, I.; Moore, H.; Lam, C.; Auer, M.; Hub, E.; Rot, A. Transcytosis and surface presentation of IL-8 by venular endothelial cells. Cell 1997, 91, 385-395.

6. Saad, O. M.; Ebel, H.; Uchimura, K.; Rosen, S. D.; Bertozzi, C. R.; Leary, J. A. Compositional profiling of heparin/heparan sulfate using mass spectrometry: Assay for specificity of a novel extracellular human endosulfatase. Glycobiology 2005, 15, 818-826.

7. Ashikari-Hada, S.; Habuchi, H.; Kariya, Y.; Itoh, N.; Reddi, A. H.; Kimata, K. Characterization of growth factor-binding structures in heparin/heparan sulfate using an octasaccharide library. J. Biol. Chem. 2004, 279, 12346-12354.

8. Kreuger, J.; Jemth, P.; Sanders-Lindberg, E.; Eliahu, L.; Ron, D.; Basilico, C.; Salmivirta, M.; Lindahl, U. Fibroblast growth factors share binding sites in heparan sulphate. Biochem. J. 2005, 389, 145-50.

9. Mohammadi, M.; Olsen, S. K. Goetz, R. A protein canyon in the FGF-FGF receptor dimer selects from an a la carte menu of heparan sulfate motifs. Curr. Opin. Struct. Biol. 2005, 15, 506-516.

10. Jin, L.; Abrahams, J. P.; Skinner, R.; Petitou, M.; Pike, R. N.; Carrell, R. W. The anticoagulant activation of antithrombin by heparin. Proc. Natl. Acad. Sci. U.S.A. 1997, 94, 4683-14688.

11. Whisstock, J. C.; Pike, R. N.; Jin, L.; Skinner R.; Pei, Y. X.; Carrell, R. W.; Lesk, R. M. Conformational changes in serpins: II. the mechanism of activation of antithrombin by heparin. J. Mol. Biol. 2000, 301, 1287-1305.

12. Shukla, D.; Liu, J.; Blaiklock, P.; Shworak, N. W.; Bai, X.; Esko, J. D.; Cohen, G. H.; Eisenberg, R. J.; Rosenberg, R. D.; Spear, P. G. A novel role for 3-O-sulfated heparan sulfate in herpes simplex virus 1 entry. Cell 1999, 99, 13-22.

13. Yu, Y.; Sweeney, M. D.; Saad, O. M.; Crown S. E.; Handel, T. M.; Leary, J. A. Chemokine-glycosaminoglycan binding: specificity for CCR2 ligand binding to highly sulfated oligosaccharides using FTICR mass spectrometry. J. Biol. Chem. 2005, 280, 32200-32208. 
14. Stringer, S. E.; Gallagher, J. T. Heparan sulphate. Int. J. Biochem. Cell Biol. 1997, 29, 709-714.

15. Stringer, S. E.; Gallagher, J. T. Specific binding of the chemokine platelet factor 4 to heparan sulfate. J. Biol. Chem. 1997, 33, 20508-20514.

16. Spillmann, D.; Witt, D.; Lindahl, U. Defining the interleukin-8-binding domain of heparan sulfate. J. Biol. Chem. 1998, 273, 15487-15493.

17. Stringer, S. E.; Forster, M. J.; Mulloy, B.; Bishop, C. R.; Graham, G. J.; Gallagher, J. T. Characterization of the binding site on heparan sulfate for macrophage inflammatory protein $1 \alpha$. Blood 2002, 100, 1543-1550.

18. Linhardt, R. J.; Turnbull, J. E.; Wang, H. M.; Loganathan D.; Gallagher, J. T. Examination of the substrate specificity of heparin and heparan sulfate lyases. Biochemistry 1990, 29, 2611-2817.

19. Yamada, S.; Murakami, T.; Tsuda, H.; Yoshida, K.; Sugahara, K. Isolation of the Porcine Heparin Tetrasaccharides with Glucuronate 2-O-Sulfate. J. Biol. Chem. 1995, 270, 8696-8705.

20. Zaia, J.; Costello, C. E. Tandem mass spectrometry of sulfated heparin-like glycosaminoglycan oligosaccharides. Anal. Chem. 2003, $75,2445-55$.

21. Laremore, N. L.; Murugensan, S.; Park, T.; Avci, F. Y.; Zagorevski, D. V.; Linhardt, R. J. Matrix-assisted laser desorption/ionization mass spectrometric analysis of uncomplexed highly sulfated oligosaccharides using ionic liquid matrices. Anal. Chem. 2006, 78, 1774-1779.

22. Behr, J. R.; Matsumoto, Y.; White, F. M.; Sasisekharan, R. Quantification of isomers from a mixture of twelve heparin and heparan sulfate disaccharides using tandem mass spectrometry. Rapid Commun. Mass Spectrom. 2005, 19, 2553-2562.
23. Henriksen, J.; Roepstorff, P.; Ringborg, L. H. Ion-pairing reversed-phase chromatography/mass spectrometry of heparin. Carbohydr. Res. 2006, $341,382-287$.

24. Hitchcock, A. M.; Costello, C. E.; Zaia, J. Glycoform quantification of chondroitin/dermatan sulfate using a liquid chromatography-tandem mass spectrometry platform. Biochemistry 2006, 45, 2350-2361.

25. Saad, O. M.; Leary, J. A. Heparin sequencing using enzymatic digestion and ESI-MS ${ }^{\mathrm{n}}$ with HOST: A heparin/HS oligosaccharide sequencing tool. Anal. Chem. 2005, 77, 5902-5911.

26. Wu, Z. L.; Lech, M.; Beeler, D. L.; Rosenburg, R. D. Determining heparan sulfate structure in the vicinity of specific sulfotransferase recognition by mass spectrometry. J. Biol. Chem. 2004, 279, 1861-1866.

27. Desai, U. R.; Wang, H. M.; Linhardt, R. J. Specificity studies on the heparin lyases from flavobacterium-heparinum. Biochemistry 1993, 32, $8140-8145$.

28. Paavola, C. D.; Hemmerich, S.; Grunberger, D.; Polsky, I.; Bloom, A.; Freedman, R.; Mulkins, M.; Bhakta, S.; McCarley, D.; Wiesent, L.; Wong, B.; Jarnagin, K.; Handel, M. Monomeric monocyte chemoattractant protein-1 (MCP-1) binds and activates the MCP-1 receptor CCR2B. J. Biol. Chem. 1998, 273, 33157-65.

29. Saad, O. M; Leary, J. A. Compositional analysis and quantification of heparin and heparan sulfate by electrospray ionization ion trap mass spectrometry. Anal. Chem. 2003, 75, 2985-2995.

30. Lubkowski, J.; Bujacz, G.; Boque, L.; Domaille, P. J.; Handel, T. M.; Wlodawer, A. The structure of MCP-1 in two crystal forms provides a rare example of variable quaternary interactions. Nat. Struct. Biol. 1997, $4,64-69$. 